\title{
Practical office ultrasound for the hip surgeon: current concepts
}

\author{
Ehud Rath, ${ }^{1}$ Zachary T Sharfman, ${ }^{2}$ Eyal Amar
}

${ }^{1}$ Minimally Invasive Orthopedic Surgery Unit, Tel Aviv Sourasky Medical Center, Affiliated to Sackler Faculty of Medicine, Tel Aviv University, Tel-Aviv, Israel ${ }^{2}$ Orthopedics, Montefiore Hospital and Medical Center Bronx, New York, USA

Correspondence to Dr Eyal Amar, Minimally Invasive Orthopedic Surgery Unit, Tel Aviv Sourasky Medical Center Orthopedics Division, 6423906, Tel-Aviv, Tel-Aviv, Israel; eyalamar73@gmail.com

Received 11 October 2019 Revised 21 December 2019 Accepted 17 January 2020 Published Online First 5 March 2020

\section{SLinked}

- http://dx.doi.org/10.1136/ jisakos-2019-000308

Check for updates

(C) International Society of Arthroscopy, Knee Surgery and Orthopaedic Sports Medicine 2020. No commercial re-use. See rights and permissions. Published by BMJ.

To cite: Rath E, Sharfman ZT, Amar E. J ISAKOS

2020;5:89-97.

\section{ABSTRACT}

Ultrasound imaging allows for real-time dynamic visualisation of muscles, tendons, fascial planes and joints. This modality enhances the sensitivity of physical examination for the localisation of pathology and improves clinical diagnostic accuracy. Ultrasound improves the accuracy of targeted injections without the need for fluoroscopy. This modality is economically affordable, portable, and does not expose the patient or provider to radiation. A thorough understanding of the anatomy and biomechanics of the hip and a working knowledge of both intra-articular and extra-articular hip pathologies is essential to optimising the utility of this modality. Ultrasound-guided injections allow for real-time visualisation of both the needle and soft tissue or intra-articular target and are superior to unguided hip injections. Improved patient care can be achieved through the skilled use of ultrasound by a hip surgeon, radiologist or ultrasound technician with appropriate training.

Ultrasound technology provides a valuable dynamic diagnostic imaging tool which can be used in real time to localise structures and assist in diagnostic tests and targeted injections. Ultrasound imaging is relatively cheap, easily accessible and user-friendly. Since 2010, non-radiologists performed more ultrasound-guided procedures than radiologists in USA. $^{1}$

Ultrasound imaging provides real-time dynamic visualisation of muscles, tendons, fascial planes and joints. This modality enhances the sensitivity of physical examination for the localisation of pathology and improves clinical diagnostic accuracy. Ultrasound improves the accuracy of targeted injections without the need for fluoroscopy. Furthermore, ultrasound imaging is economically affordable and portable, and does not expose the patient or provider to ionising radiation. Ultrasound image acquisition is highly operator-dependent. Experienced technicians, radiologists and orthopaedic surgeons with in-depth knowledge of the physical attributes of ultrasound waves, image generation techniques and anatomical structures can successfully use this technology to diagnose a variety of pathologies while recognising imaging artefacts and preventing missed or misdiagnoses.

\section{BASICS OF ULTRASOUND}

Ultrasound technology uses the piezoelectric effect, to generate sound waves from electric current applied to crystals within the ultrasound transducer. These waves are then transmitted through the ultrasound gel and rebound on anatomical structures to finally be absorbed as sound waves, and subsequently are transformed to electric signals and finally images. ${ }^{2}$

Transducer frequency determines depth of penetration and image resolution. In general, higherfrequency ultrasound waves (short wavelength) generate higher resolution images with less penetration into anatomical structures, while lowerfrequency ultrasound waves (long wavelength) generate lower resolution images but can penetrate into deeper structures secondary to a lower degree of attenuation. ${ }^{3}$

It is important to choose the optimal frequency to improve visualisation depending on the depth of a structure. High-frequency transducers (10-15 $\mathrm{MHz}$ ) are optimal for the imaging of superficial structures such as patellar tendon, rotator cuff or Achilles tendon and low-frequency transducers (1-5 MHz) are optimal for imaging the anatomy of the hip and deep muscular structures (table 1).

Acoustic impedance is defined as an object's resistance to the movement of a wave or the quantity of echo returned after striking a tissue interface. The angle of incidence is defined as the angle between the sonographic wave and the tissue it is interacting with. Both acoustic impedance and the angle of incidence influence the image rendered by the sonograph. One example of how these principal effect ultrasound imaging occurs is when the ultrasound beam is incident on fibrillar structures such as a tendon or a ligament. The organisation of the fibrils in parallel can reflect the majority of the sound wave away from the transducer resulting in an interpretation of an hypoechoic artefact. This phenomenon is termed 'anisotropy artefact'. This can result in missed or misdiagnosis of tendinosis or tendon tear (figure 1).

\section{SONOGRAPHIC NORMAL HIP ANATOMY}

No universal protocol exists for the sonographic evaluation of body regions. ${ }^{4}$ When a provider is approaching the hip they should use a systematic approach to scanning which is both thorough and reproducible. Furthermore, as sonographic assessment is a dynamic tool it may be best used as an extension of the physical examination. Ultrasound evaluation of the hip should thus be performed in accordance with the patient's clinical presentation and differential diagnosis. For example, simply positioning the ultrasound probe over the region of maximal tenderness may illuminate pathological swelling, fluid or tendon tears in the underlying anatomical structures (whether intra-articular or extra-articular). Moreover, ultrasound enables the provider to assess deep structures which are not readily palpable in physical examination such as the 


\section{Current Concepts}

\section{Pearls}

- Ultrasound provides real-time visualisation of muscles, tendons, fascial planes and joints. It offers improved sensitivity for clinical localisation of pathology and improves the accuracy of diagnoses.

- Transducer frequency determines depth of penetration and image resolution. High-frequency ultrasound waves (short wavelength) generate high resolution images for superficial structures, while low-frequency ultrasound waves (long wavelength) generate lower resolution images and are best used for deeper structures.

- One should have a systematic approach to scanning that serves as a thorough and reproducible demonstration of the assessed region.

- A thorough understanding of the anatomy and biomechanics of all five layers of the hip, that is the osseous, capsulolabral, musculotendinous, neurovascular and kinematic chain, is required.

- Ultrasound-guided hip joint injections are significantly more accurate than landmark-guided intra-articular hip injections.

- An inplane approach should be used around the pelvis/hips to visualise the entire needle at all times.

- The needle should be kept as perpendicular as possible to the sound beam.

- Improved quality of care for patients can be achieved through skilled use of ultrasound by a hip surgeon with appropriate training.

\section{Indication for office sonographic evaluation of the hip}

- Localisation of pathology.

- Unclear source of pain: improves the accuracy of diagnoses (lidocaine test).

- Injections to the target site with no need for fluoroscopy.

- Dynamic imaging of motion-associated pathology.

\section{Tips and tricks}

- Prevent anisotropy: aim the beam perpendicular to the long axis of the insonated structure.

- Place the transducer in a longitudinal orientation to the insonated structure.

- Use the location of the transducer at the point of maximal tenderness to assess the anatomical structure involved with the clinical symptoms.

- Safe injection: the entry point should allow maximal specular reflection of the needle aiming the probe in the long axis (needle parallel to the transducer). The position of the tip of the needle must be clearly seen before starting the injection.

\section{Major pitfalls of hip ultrasound}

- Ultrasound is operator dependent with a long learning curve to provide expertise in both normal and abnormal findings.

- Inadequate angulation of the probe relative to the insonated structure may result in anisotropy depicted as an area of decreased echogenicity of the evaluated structure thus misinterpreted as a tear.

- Technology innovation in the area of diagnostic ultrasound imaging has been quite dynamic. Solutions considered high end 2 years ago are currently considered mid-range, or even in some cases, considered low end options.

\section{Basics of Ultrasound}

Sonographic normal hip anatomy

- Basic scanning principles and normal tissue appearance

- Anterior Joint Space and Iliopsoas Tendon

- Medial Hip

- Lateral Hip

- Posterior hip

- Sonographic normal hip anatomy

\section{Pathologies}

- Anterior

- Subspinal Impingement (SSI)

- Osteoarthritis (OA)

- Effusion

- Iliopsoas tendinitis and bursitis

- Lateral Hip Pathology

- Posterior

- Deep gluteal space

\section{Ultrasound guided injections}

- Benefit of US guided hip injections or aspiration

- Principles of injections

- Technique and accuracy of US guided injections

- Intra articular injection

- lliopsoas Bursa 
Current Concepts Continued

- Ultrasound-Guided Greater Trochanteric Bursa Injection

- Piriformis

- Ultrasound-Guided Ischial Bursal Injection

- Perisciatic

Limitations

Future Perspectives of Hip Ultrasound

iliopsoas tendon and to evaluate the structure dynamically while correlating its movement with a patient's physical complaints.

\section{BASIC SCANNING PRINCIPLES AND NORMAL TISSUE APPEARANCE}

Different tissues have unique sonographic presentations. Bony structures typically appear as hyperechoic smooth lines or circles (depending on the orientation of the transducer) that cast acoustic shadows. Articular cartilage typically presents a hypoechoic (black) band secondary to high fluid content in the cartilage. ${ }^{5}$ Longitudinally, muscles typically have a pennate appearance, however in the transverse orientation muscles typically will have a speckled appearance. Tendons and nerves are typically hyperechoic.

\section{Scanning technique of anterior hip: anterior joint space and iliopsoas tendon}

With the patient positioned supine, the transducer can be placed in an oblique longitudinal plane over the femoral neck. This starting position can help to establish easily recognisable landmarks such as the femoral head and the acetabulum (figure 2). In this position, the anterior labrum, which is difficult to demonstrate, appears as a homogeneously hyperechoic triangular structure. The femoral triangle is located anteromedially in the upper third of the thigh containing the femoral vein, artery and nerve, oriented from medial to lateral. Lateral to the neurovascular bundle, the iliopsoas muscle can be identified. The iliopsoas tendon arises from the muscle in a deep and eccentric manner within a posterior medial aspect of the muscle, and lays just anterior to the joint capsule (figure 3). The anterior inferior iliac spine (AIIS) is visualised just lateral to the iliopsoas, and just proximal to the joint line. The iliocapsularis can be seen originating from the inferior aspect of the AIIS and the direct head of the rectus femoris tendon can be seen originating from the superior aspect ${ }^{6}$ (figure 4).

On longitudinally oriented imaging, the joint capsule is seen as a hyperechoic or isoechoic band just superficial to the hypoechoic bone. The distance between the femoral neck to the outer margin of the fibrous capsule in adults ranges from $4 \mathrm{~mm}$ to $9 \mathrm{~mm}$ (mean, $6.4 \mathrm{~mm}$ ), and does not vary with respect to body morphology or age ${ }^{78}$ (figure 2). One common finding on hip

\section{Future perspectives}

Future advancements in technology will improve the diagnostic accuracy and interventional potential of ultrasound imaging. Enhanced image reconstruction and display will provide a noninvasive modality for assessment of the hip in static and dynamic automated imaging techniques. Improved sonographic imaging will provide more accurate office diagnosis of current MRI-based diagnoses. ultrasound is an effusion which is defined as capsular distension greater than $5 \mathrm{~mm}$ or a dissimilarity of greater than $1 \mathrm{~mm}$ between the affected hip and the contralateral hip (figure 5).

\section{Medial hip}

Examination of the medial hip and surrounding musculature is important as these structures are common sites for muscle or tendon strains. The patient can be positioned supine with the thigh abducted and externally rotated. Knee flexion will help to facilitate this position. The insertion of the iliopsoas tendon on the lesser trochanter is a good landmark to help identify structures and can be seen clearly in the long axis plane. The muscular planes of the adductors can be recognised in the medial leg including: the superficial layer comprised of the adductor longus laterally and the gracilis medially, the adductor brevis in the middle layer, and finally the adductor magnus in the deep layer. Scanning cranially in the long-axis a provider can follow these muscles up to their origin on the pubis. Specifically, the adductor longus tendon origin is visualised as a triangular hypoechoic shape.

\section{Lateral hip}

Lateral hip pain is a common complaint and ultrasound of the peritrochanteric region can help to elucidate a source for this pain. The gluteal muscles, greater trochanter, trochanteric bursa and iliotibial band are easily visualised by ultrasound. ${ }^{9}{ }^{10}$ With the patient placed in the lateral decubitus position, a transducer can be placed over the trochanter. In a non-pathological exam, the bursae around the greater trochanter are not visible as their fluid content is too small. Cranially and posteriorly, the anterior margin of the gluteus medius (superficial) and gluteus minimus (deep) comes into view (figure 1A). Coronally oriented images will demonstrate the fascia latae as a superficial hyperechoic band overlying the gluteus medius muscle, tendon and the greater trochanter.

\section{Posterior hip}

Posterior hip pain can emanate from various pathologies including neurological compression (eg, sciatic), muscular pathology (eg, proximal hamstrings) or bony impingement (eg, ischiofemoral) source. To assess the posterior hip the patient is positioned prone. The ischial tuberosity can be palpated in the lower part of the buttock and the origin of the semimembranosus,

Table 1 Typical features of transducer frequency

\begin{tabular}{|c|c|c|}
\hline $\begin{array}{l}\text { Transducer } \\
\text { frequency }\end{array}$ & Low (1-5 MHz) & High (10-15 MHz) \\
\hline Penetration & Deep & Superficial \\
\hline Structures & Intracapsular, sciatic nerve & $\begin{array}{l}\text { AllS, adductors, } \\
\text { peritrochanteric region }\end{array}$ \\
\hline
\end{tabular}

AllS, anterior inferior iliac spine. 

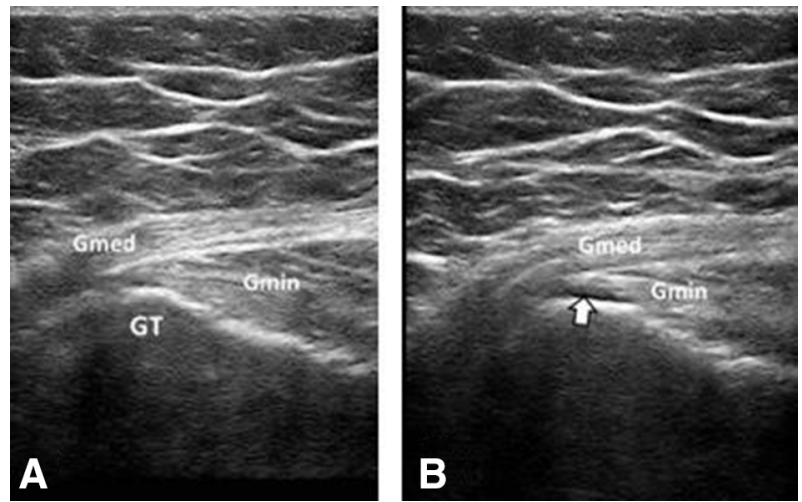

Figure 1 Anisotropy: a tilt of the transducer produces an artefact that may prompt an incorrect diagnosis of a tendon tear. (A) Normal appearance of abductor muscle of the hip. (B) Appearance of black stripe (arrow) can be erroneously interpreted as abductor tear. Gmed, gluteus medius; Gmin, gluteus minimus; GT, greater trochanter.

semitendinosus, long head of the biceps femoris muscles can be easily recognised in the posterior axial plane. ${ }^{11-13}$ Pathological fluid in this region can denote a muscle tear or avulsion. In the prone position the sciatic nerve can be seen lateral and deep (anterior) to the semimembranosus, semitendinosus and biceps femoris as a flattened tubular hyperechoic structure, $5-9 \mathrm{~mm}$ in diameter (figure 6). ${ }^{14}$

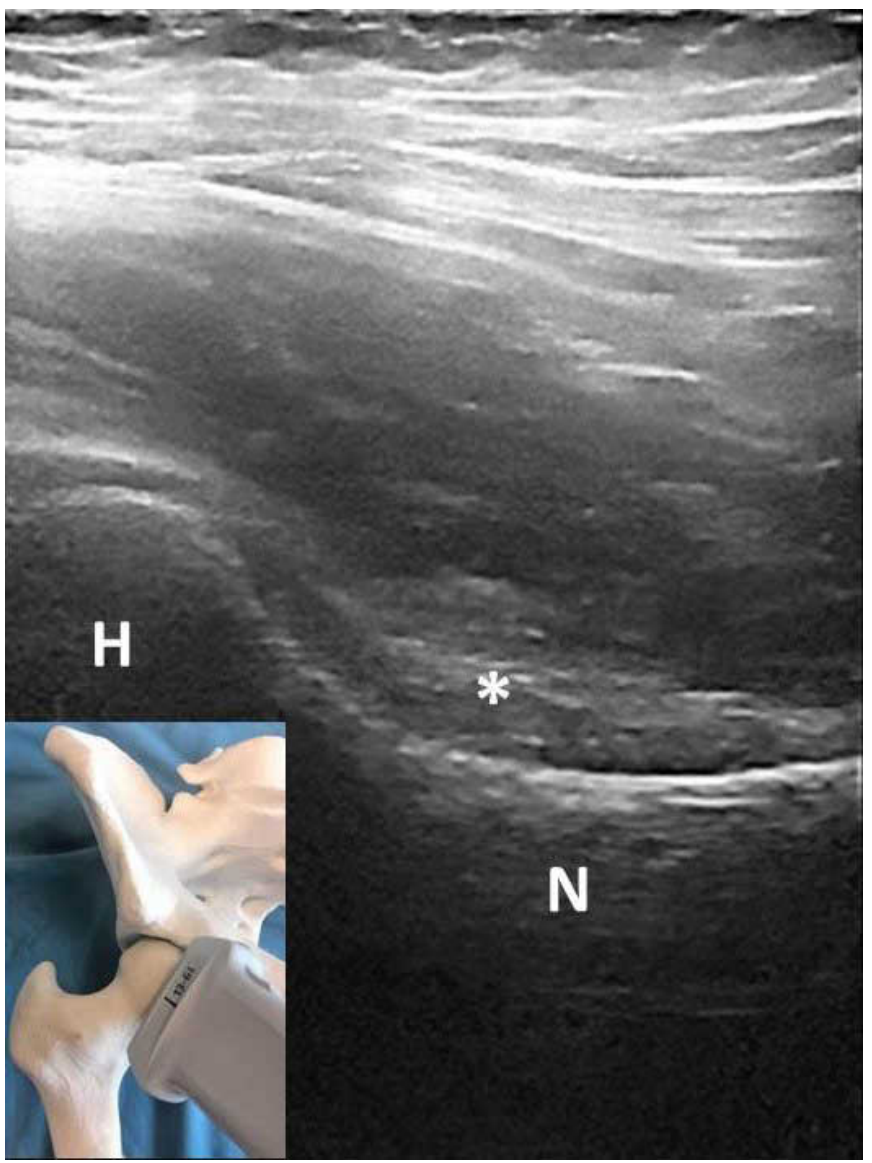

Figure 2 The transducer is placed in an oblique longitudinal plane. $\mathrm{H}_{\text {, }}$ femoral head; $\mathrm{N}$, femoral neck; ${ }^{*}$, capsule.

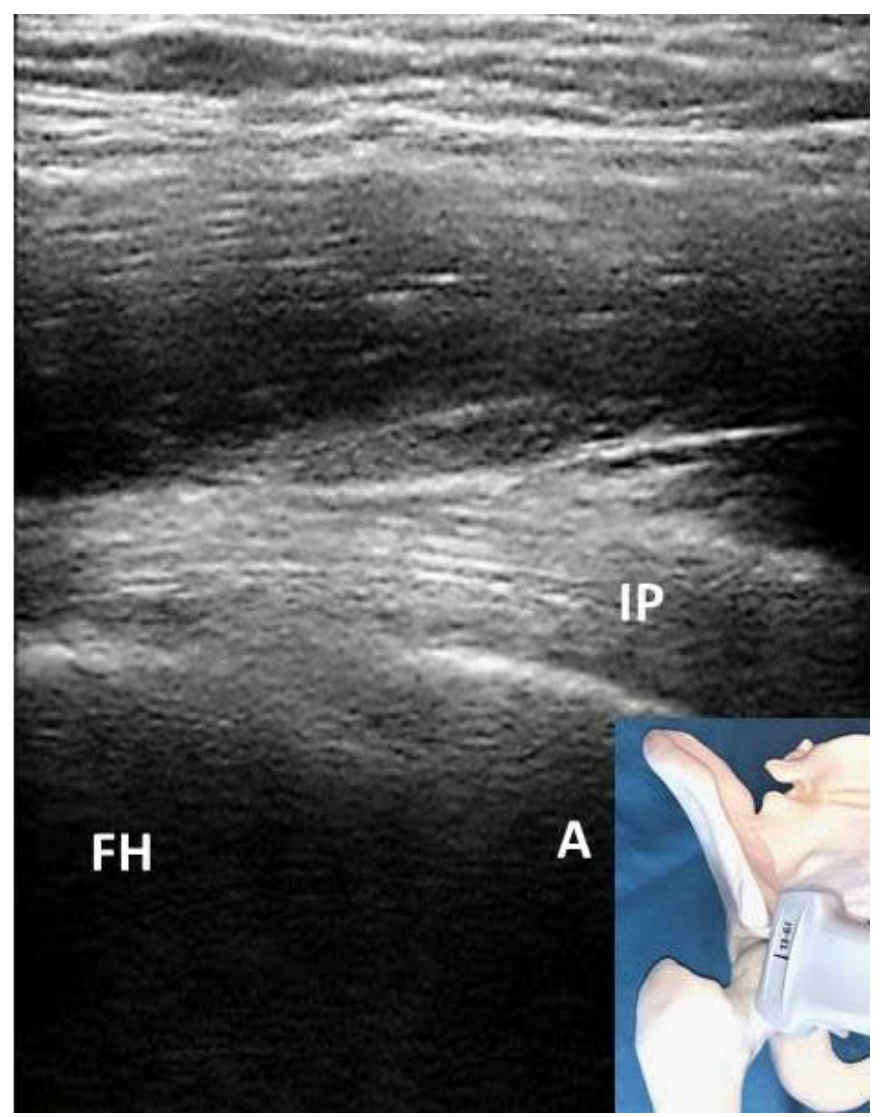

Figure 3 The transducer is placed in the longitudinal plane of the iliopsoas tendon. A, acetabulum; FH, femoral head; IP, iliopsoas tendon.

\section{PATHOLOGIES}

The pelvis and hip are comprised of complex dynamic anatomical structures. This complexity demands a systemic approach to clinical and ultrasound evaluation. A thorough understanding of the anatomy and biomechanics of the hip in terms of the osseous, musculotendinous, capsulolabral, neurovascular structures as well as the kinematic chain is necessary. Understanding of the aetiology of pathologies in the hip region and the current research describing common intra-articular and extra-articular hip problems is essential to understanding and diagnosing the vast spectrum of hip pathologies.

\section{Anterior}

Femoroacetabular impingement (FAI) clinical presentation, imaging findings and intraoperative signs have been well described. ${ }^{15}$ Imaging of CAM-type FAI demonstrates a nonspherical femoral head specifically in the anterior and anterolateral aspect of the head-neck junction. Plain radiographs are typically the first imaging modality obtained for patients with complaints of hip pain but ultrasound can be used to detect the presence of a non-spherical head-neck junction. ${ }^{16}$ This can be reliably achieved with ultrasound imaging with the hip in $20^{\circ}$ internal rotation. This technique has been demonstrated to be as reliable as plain frog-view radiographs in determining the alpha angle and offset ratio for the diagnosis of CAM-type FAI. ${ }^{17}$

Robinson e $_{\text {al }}{ }^{18}$ assessed the discrepancy between CT measurement of alpha angle and ultrasound calculation of alpha angles and found it to be slightly more than $10^{\circ}$. Ultrasound showed sensitivity of $91.3 \%$ and specificity of $43.75 \%$ when compared with CT. Extra-articular causes of impingement have also been identified to produce pain and limited function especially among 


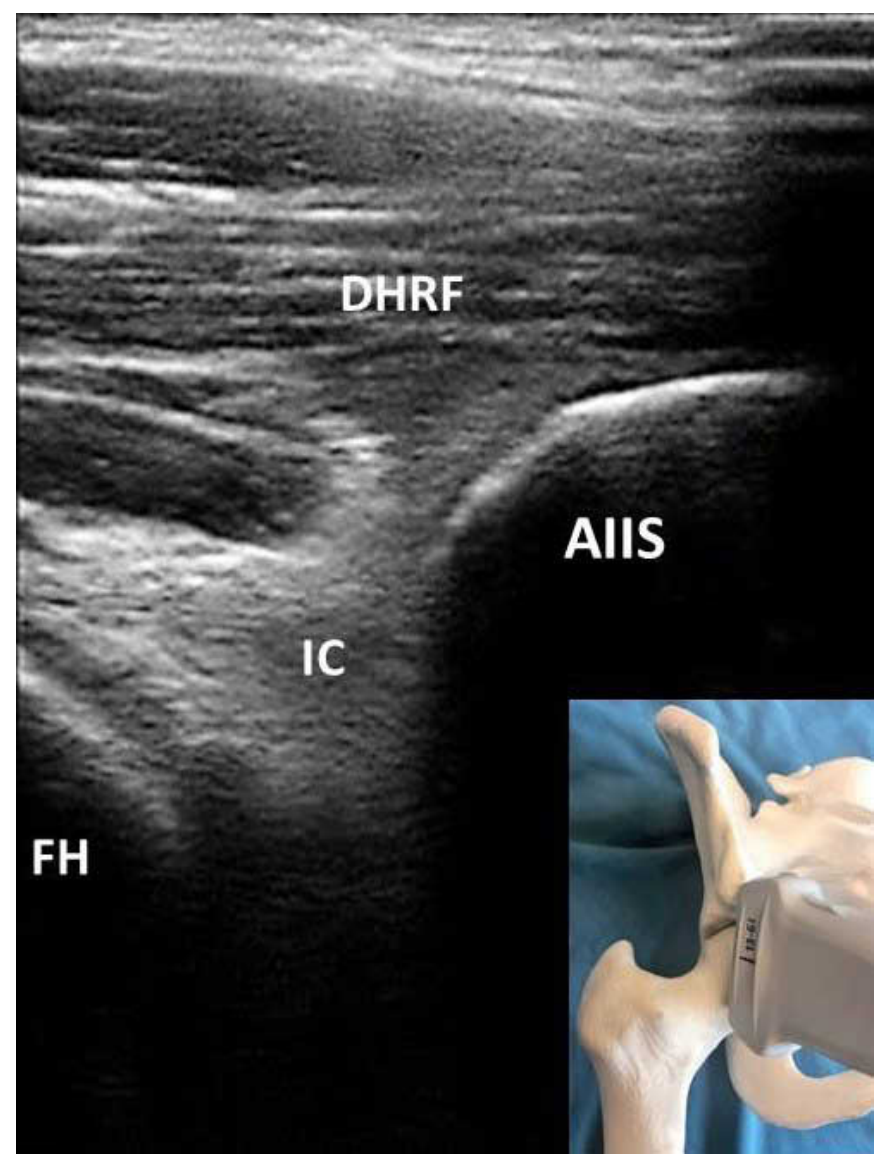

Figure 4 The transducer is placed in the longitudinal plane demonstrating both the AlIS and the femoral head. AllS, anterior inferior Iliac spine; DHRF, direct head of the rectus femoris; FH, femoral head; IC, iliocapsularis.

young patients. Sources for extra-articular impingement include psoas impingement, subspinal impingement (SSI), greater trochanteric/pelvic impingement and ischiofemoral impingement (IFI). ${ }^{19}$ These structures can be assessed dynamically with ultrasound to look for impingement.

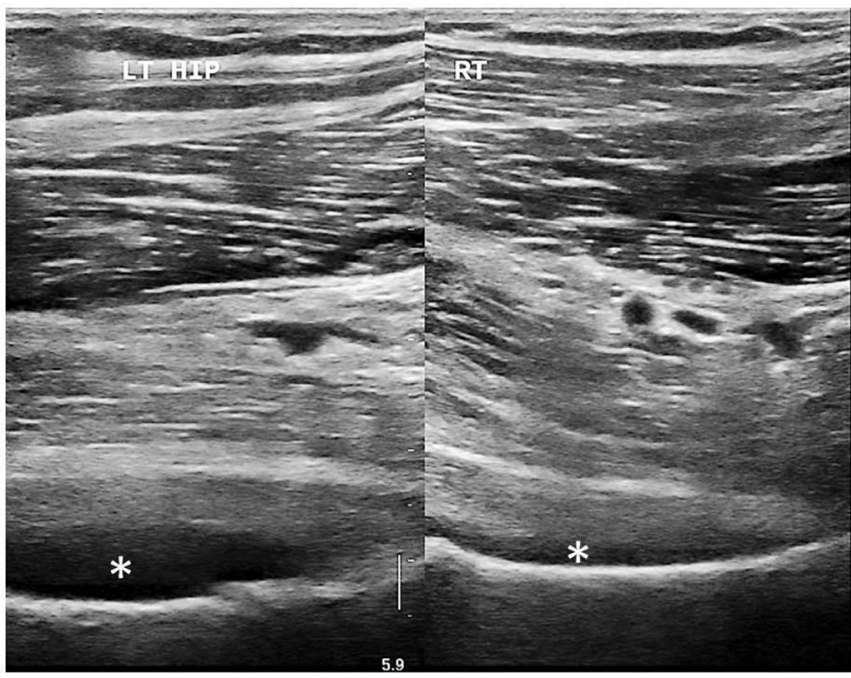

Figure 5 Left hip effusion (asterisk) indicated by more than a $1 \mathrm{~mm}$ difference in capsular distension between both hips (LT-Left, RT-Right)
Subspinal impingement

SSI is a relatively novel clinical entity. This pathology presents with anterior groin pain, pain with hip flexion and limitations in range of motion. SSI is caused by a low or morphologically abnormal AIIS which causes extra-articular impingement with the femur. ${ }^{20}$ Plain anteroposterior (AP) radiography underestimates the prevalence of low AIIS. ${ }^{21}$ A morphological type II or type III AIIS has a low vertical distance from the AIIS to the acetabular rim. ${ }^{22}$ Traditional AP radiographs superimpose the AIIS over other bony structures but a false profile view allows for visualisation of the AIIS and its relationship to the acetabular rim.

The AIIS can be visualised sonographically (figure 4). A type I AIIS can be distinguished from either a type II and type III AIIS by the presence of a flat bony surface between the acetabular rim and the distal aspect of the AIIS. ${ }^{22}$ AIIS type II and type III are associated with SSI which can be demonstrated by dynamic ultrasound evaluation confirming impingement between a low-lying AIIS and the femur.

\section{Osteoarthritis}

Plain radiographs are the standard imaging modality used to evaluate osteoarthritis (OA). Despite this, sonography can highlight various anatomical structures in exceptional detail in a trained hand and detect hallmark signs of OA. Ultrasound is

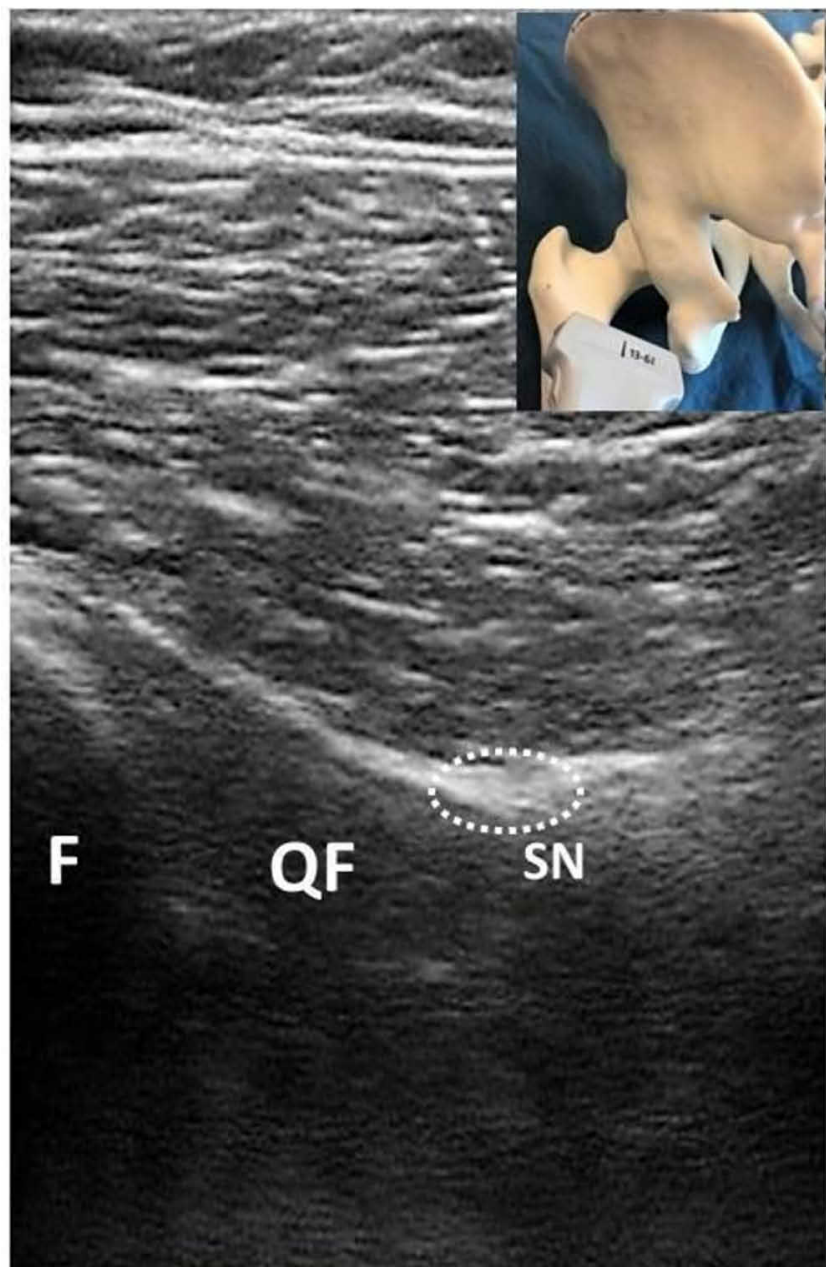

Figure 6 A transverse view at the level of the Lesser tuberosity and Ischial tuberosity: The sciatic nerve is seen as a flattened structure between the Gluteus maximus and Quadratus femoris. (F-Femur, QF-Quadratus Femoris, SN-Sciatic Nerve) 
likely a better modality for detecting joint effusion than plan radiographs and ultrasound can evidence osteophytes, articular cartilage lesions, bony cortex as well as synovial thickening and hypertrophy. Ultrasound provides a reproducible, cheap and radiation-free tool with which to assess for degenerative joint changes and changes related to synovial inflammation. ${ }^{23}$

\section{Effusion}

In the hip joint an effusion is defined as capsular distension of more than $5 \mathrm{~mm}$ or more than a $1 \mathrm{~mm}$ difference between hips (figure 5). ${ }^{24} \mathrm{~A} 1 \mathrm{~mm}$ difference in capsular distention has been shown to be more clinically correlated with patient symptoms. ${ }^{25}$ Providers using ultrasound must be aware that synovial thickening can mimic an effusion and has limited utility in diagnosis of intra-articular pathology, such as labral tear or avascular necrosis. ${ }^{26}$ However if an effusion is present, intracapsular free bodies and synovial pathologies can be readily demonstrated.

\section{Iliopsoas tendinitis and bursitis}

Inflammation of the iliopsoas tendon and/or bursa is associated with snapping hip syndrome. Whether this pathology is due to a prominent acetabular component or an inflamed tendon/bursa, it can be extremely troublesome for patients. Bursitis is visualised as a hypoechoic collection on ultrasound. A detailed history may help to establish a high index of suspicion for coxa saltans (snapping hip syndrome), and a thorough clinical examination can help to localise internal or external causes. Moreover, clinical examination, in which ultrasound can help to visualise the pathology and to guide lidocaine injection, can provide further evidence of the source of pain. This can then help to guide conservative interventions such as ultrasound-guided steroid injection.

In patients who can voluntarily reproduce the snap, the iliopsoas tendon can be sonographically visualised as it creates the snap during range of motion. ${ }^{27}$ This study can be performed by positioning the patient supine with the contralateral leg held in hip and knee flexion to expose the groin region of the affected limb. The snapping can be reproduced by guiding the leg from hyperflexion, abduction and external rotation into extension, adduction and internal rotation. Additionally, ultrasound images comparing the affected and unaffected side may demonstrate that the affected iliopsoas tendon is found in a location more medial than the unaffected iliopsoas when the patient in a supine position. ${ }^{28}$

\section{Lateral hip pathology}

Lateral hip pathologies are readily demonstrated during ultrasound of the soft tissue of the hip. Trochanteric bursitis can be easily identified on ultrasound by the presence of anechoic or hypoechoic fluid on lateral hip ultrasound. Typically, the greater trochanteric bursa is found just posterior to the posterior facet of the greater trochanter. When scanning over the gluteus medius or minimus, a thickened heterogenous tendon with loss of the normal fibrillar pattern is characteristic of abductor tendinosis, and calcifications within the tendon are also commonly seen. ${ }^{11-13}$ Partial or full-thickness abductor tears can be visualised as interruptions in the fibrillar pattern of the abductor tendons. Other characteristic signs of these tears include fluid about the tendon or decreased tendon mass.

Fusiform enlargement of the iliotibial band about the greater trochanter is a sign of tendonitis and an anechoic defect within the band may represent a tear. ${ }^{29}$ These clinical pathological signs are often seen with external snapping hip syndrome (coxa saltans externa) and can be demonstrated in transverse and longitudinal scanning of the lateral hip. Displacement of the iliotibial band over the greater trochanter can be seen when a patient voluntarily reproduces the snap, confirming the diagnosis of external snapping hip. $^{27}$

\section{Posterior}

\section{Deep gluteal space}

The dimensions of the ischiofemoral space are largely dependent on hip positioning. ${ }^{30}$ With the hip held in abduction and internal rotation the space is largest, whereas adduction and external rotation decrease the space to its smallest dimensions. To examine this space, the patient is positioned prone, with the knee flexed at $90^{\circ}$ to control femoral rotation. In the axial plane, the medial aspect of the lesser trochanter and the lateral aspect of the ischial tuberosity are identified. Then the transducer can be angled medially to visualise the ischiofemoral space. The narrowest interval between these landmarks can be measured using an ultrasound. When this space is measured to be less then $1.5 \mathrm{~cm}$ it is suggestive of IFI. ${ }^{31}$

\section{ULTRASOUND-GUIDED INJECTIONS Benefit of ultrasound-guided hip injections or aspiration}

Fluoroscopic and sonographic guidance have become a commonplace targeting technique to aid in hip injections and aspirations. A good understanding of hip anatomy and neurovascular structures is necessary to ensure safe hip injections and aspirations. Especially as non-radiologists are performing more ultrasoundguided procedures, ensuring proper training is essential for patient safety. ${ }^{1}$

Hip joint injections are technically challenging as the hip joint is a deep structure which is very congruent. Furthermore, the provider has to avoid the various neurovascular bundles around the hip to safely perform this procedure. ${ }^{32}$ Hip injections, without guidance, are reported to enter the joint only 51\%-93\% of the times. ${ }^{33}$ Furthermore, these attempts may pass close to neurovascular structures or even penetrate them. Ultrasound facilitates real-time visualisation of the needle and target, which can improve outcomes.

\section{Principles of injections}

Ultrasound-guided injections should begin with an ultrasonography examination to identify relevant landmarks and the injection target. Doppler images may be used to identify and avoid vessels. For example, the femoral artery must be avoided in iliopsoas or hip joint injections, and the superior/inferior gluteal arteries should be identified and avoided in piriformis injections. We recommend an inplane approach for ultrasound-guided injections around the pelvis/hips. This assists the provider in visualising the needle path during the procedure. Once the anatomical landmarks are identified and the probe position is confirmed, the injection site can be prepped in the standard sterile fashion and local anaesthesia can be administered. An appropriate length and gauge needle can then be advanced to the targeted position and aspiration can be followed by injection. Maintaining the needle in an orientation perpendicular to the ultrasound beam will ensure that the needle is echogenic and easily identifiable. Some manufacturers produce needles with specific design features that enhance their echogenicity; this may assist especially in deep-targeted injections or areas with limited access. ${ }^{34}$ Additionally, as ultrasound is a dynamic image, oscillating the needle gently while slowly scanning with the transducer can help to locate the needle tip in difficult situations. ${ }^{35} 36$

\section{Technique and accuracy of ultrasound-guided injections Intra-articular injection}

Hip joint injections are technically challenging. The hip joint is a deep structure, patients have variable body habitus, acetabular 


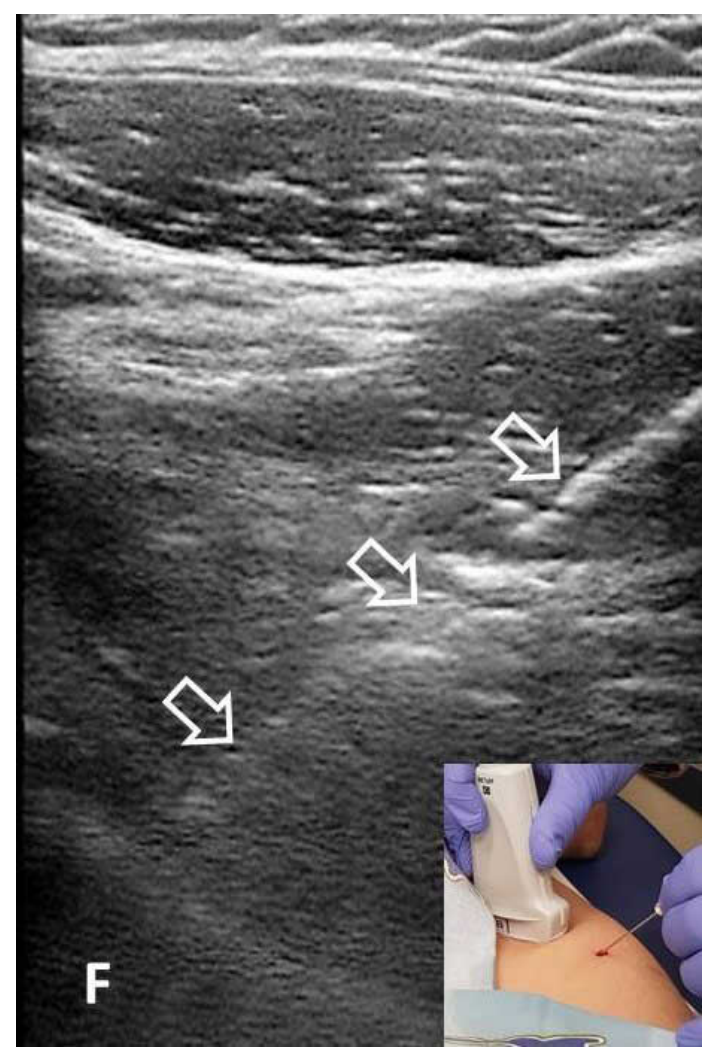

Figure 7 Ultrasound-guided injection into the anterior joint recess at the junction of the femoral head and neck. F, femur; arrows, needle.

morphology can greatly alter access to the joint and the adjacent femoral neurovascular bundle lies close to the target. Surface landmark-guided hip joint injections can be performed, however ultrasound-guided hip joint injections are significantly more accurate and reproducible. ${ }^{33}$

Leopold $e t a l^{32}$ reported that the risk of piercing the femoral nerve was $27 \%$ when using the 'blind' anterior approach to hip injections. Furthermore, the authors reported that the needle tips passed within $5 \mathrm{~mm}$ of the femoral nerve in $60 \%$ of cases.

Yoong et $a l^{37}$ evaluated the utility of ultrasound-guided hip joint injection in predicting surgical outcomes. The authors found that $93 \%$ of patients who experienced pain relief after injection achieved a successful surgical outcome, whereas only $63 \%$ of patients with partial relief achieved such an outcome. Moreover, 44 of $49(89.8 \%)$ patients who did not experience any relief from the injection ultimately did not undergo hip surgery.

\section{Technique}

Using an inplane approach, the transducer is placed in the oblique-sagittal plane, and the hip joint and relevant structures are identified. Marking the transducer position prior to skin prep can be helpful. Sterile skin preparation is performed and local anaesthetic is delivered. Using a 22-gauge 64-89 mm needle, the needle is advanced under direct visualisation into the anterior joint recess. This is found at the junction of the femoral neck and head (figure 7). An experienced hand can feel the needle penetrating the hip joint capsule. Ultrasound guidance may demonstrate flow as the injectate is delivered, and distention of the capsule may be seen. ${ }^{38}$ If the purpose of the procedure is to assess for pain, it is important to wait for a minimum of $10 \mathrm{~min}$ if lidocaine is used and $45 \mathrm{~min}$ if bupivacaine is used prior to assessing pain relief as these medications may display delayed onset of their anaesthetic effect.

\section{Iliopsoas bursa}

The iliopsoas muscle and tendon are strong muscles that produce hip flexion. This motion subjects the tendon to high mechanical stress in a confined area. The iliopsoas bursa can become inflamed and cause pain or discomfort. Ultrasoundguided iliopsoas bursa injections can provide diagnostic evidence of pathology or even provide symptomatic treatment. Positive response to the diagnostic injection may predict favourable surgical outcomes for iliopsoas tendon release in the setting of snapping iliopsoas tendon. ${ }^{39}$

\section{Technique}

The patient is positioned supine with the hip in neutral rotation. A medium-frequency or low-frequency linear-array transducer is used and positioned over the femoral head, in the transverse plane. The transducer is then slid superiorly and oriented parallel to the inguinal ligament. When snapping iliopsoas tendon is suspected, dynamic examination is performed as the hip is brought from flexion, abduction, external rotation into adduction, internal rotation and extension to reproduce the pathology. ${ }^{39}$ The transducer position can be marked and then the site is prepped in the usual sterile fashion. After administration of local anaesthetic, using an inplane technique, the needle is advanced to the deep lateral portion of the iliopsoas tendon. Hydrodissection (separation of tissue layers using fluid) can be used to identify the plane between the hip capsule and iliopsoas tendon. ${ }^{38}$ Once in position the injectate can be delivered.

\section{Ultrasound-guided greater trochanteric bursa injection}

Corticosteroid and local anaesthetic delivered into the trochanteric bursa can provide prolonged relief for patients with trochanteric bursitis. ${ }^{40}$ Several techniques exist for performing this procedure including surface landmark techniques, ${ }^{10} 40$ fluoroscopic techniques ${ }^{41}$ and ultrasound-guided techniques. ${ }^{42}$

\section{Technique}

With the patient in the lateral decubitus position, the hips and knees are flexed. The transducer is positioned in the transverse plane over the proximal femur. Identification of the gluteus minimus and medius tendons over the anterior and lateral facets, respectively, confirms positioning. Distention of the greater trochanteric bursae may be seen. With the transducer in the transverse plane over the trochanteric bursa, the skin is prepped in the usual sterile fashion. Using the inplane technique, the needle is advanced between the gluteus maximus/iliotibial band and the deep gluteus medius tendon, and the anaesthetic is then delivered. ${ }^{38}$

\section{Piriformis}

Piriformis syndrome is characterised by extrapelvic sciatic nerve compression. Patients may present with symptoms typical of sciatica that can be reproduced with flexion adduction internal rotation. Piriformis syndrome is considered a diagnosis of exclusion but guided injections may help establish or confirm a suspected diagnosis. Surface landmark-guided piriformis injections are difficult and guidance is recommended to improve safety and accuracy. Finnoff et $a l^{43}$ reported that ultrasoundguided piriformis injections were 95\% accurate as compared with fluoroscopy-guided injections which were only $30 \%$ accurate. Conversely, Fowler et al reported that patient satisfaction, 
pain scores and procedure time were not significantly different between ultrasound-guided and fluoroscopically guided piriformis injections. ${ }^{44}$

\section{Technique}

The patient is positioned prone and the transducer is placed over the posterior superior iliac spine (PIIS) in the axial plane. Scanning inferiorly, the transducer is moved until the lateral sacrum and PIIS appear as medial and lateral structures, respectively. The transducer is then scanned inferior to the PIIS so the ilium disappears from the image, as the greater sciatic notch is visualised. The piriformis is then seen in the long axis travelling superomedial to inferolateral just deep to the gluteus maximus muscle. Unless the patient has variant anatomy, the sciatic nerve will be located deep to the piriformis muscle. The transducer is maintained in a position parallel to the piriformis muscle and the position is marked. The area is then prepped in the standard sterile fashion. Using the inplane technique and a lateralto-medial approach, the needle is advanced through the gluteus maximus, until the piriformis muscle is entered. The injectate is then delivered. ${ }^{38}$

\section{Ultrasound-guided ischial bursa injection}

Injection of corticosteroids to the ischial bursa has been shown to provide relief to patients when conservative treatments of ischial-gluteal bursitis have been unsuccessful. ${ }^{45} 46$ Cadaveric study of ultrasound-guided ischial bursa injection has shown that ultrasound-guided ischial bursa injections produce accurate ischial bursograms without overflow or endangering surrounding neurovascular structures. ${ }^{45}$ The distance between the sciatic nerve and the ischial tuberosity changes dramatically in the prone and lateral positions. When prone, the mean distance between the nerve and ischial tuberosity is reported to be $28.4 \mathrm{~mm}$ (range, $20.5-38.9 \mathrm{~mm}$ ) whereas when positioned lateral with the hip flexed to $90^{\circ}$ the mean distance increases to $41.9 \mathrm{~mm}$ (range, $30.9-66.0 \mathrm{~mm}$ ). ${ }^{45}$ The lateral decubitus position with hip flexion may decrease iatrogenic injury to the sciatic nerve during ischial bursa injection.

\section{Injection technique}

The patient is positioned lateral with the ipsilateral hip flexed to $90^{\circ} .{ }^{45}$ The transducer is positioned transversely over the ischial tuberosity. Scanning laterally with the transducer, the sciatic nerve is seen superficial to the quadratus femoris muscle. ${ }^{45}$ For safe injection, an image simultaneously demonstrating the gluteus maximus muscle, hamstring tendons, ischial tuberosity and sciatic nerve should be obtained. Once this image is achieved, the transducer position can be marked (in order to quickly return to the same spot) and the skin is prepped in the standard sterile fashion. Using the inplane technique and in a lateral-to-medial position, the needle is advanced into the region of the ischial bursa, located deep into the gluteus maximus and superficial to the hamstring tendons, and the injectate is delivered.

\section{Perisciatic}

Nwawka $e t a l^{47}$ reported on the safety and efficacy of ultrasoundguided subgluteal sciatic nerve injections. The indications for injection were either diagnostic or therapeutic for sciatica. The authors demonstrated that in a sample of 221 ultrasound-guided subgluteal sciatic nerve injections, they achieved $100 \%$ success without complications. Of the patients with follow-up data, 68\% reported symptomatic improvement. The authors discussed that patients who reported no relief had suboptimal preprocedure screening.

\section{Technique}

With the patient in the prone position the nerve can be seen as a hyperechoic tubular structure between the long head of the biceps femoris muscle laterally and the semimembranosus muscle medially, lying on the adductor magnus. Scanning proximally the nerve is followed until it reaches the plane between the gluteus maximus and short external rotators. The transducer position can be marked and the injection site is prepped in the standard sterile fashion. A needle is then introduced and advanced using an inplane technique. Ideally, the transducer is maintained at a $45^{\circ}$ angle relative to the skin in the longitudinal plane with an inclination of $5^{\circ}$ laterally. Aiming slightly lateral to medial, the needle is advanced until it reaches the level of the sciatic nerve but is in a position immediately medial to the nerve, in the muscle-aponeurotic plane. The injectate is then delivered. ${ }^{48}$

\section{Limitations}

The ultrasound is an operator-dependent modality. In novice users, both technical and visual limitations (understanding threedimensional anatomy on a two-dimensional plane) are significant barriers to effective use. Significant practical training is necessary to develop proficiency and the ability to assess abnormal anatomy with ultrasound technology. There is a tendency to overdiagnose pathology when using ultrasound. This can potentially result in unnecessary medical or surgical interventions. As such, proper training in ultrasound, performing dynamic examination, and correlating between clinal and sonographic findings is important to ensure proper use.

\section{Future perspectives of hip ultrasound}

Technological advances continue to improve image quality and instrument size in ultrasound. Ultrasound technology is now available as personal portable devices that can be carried by individual healthcare providers in clinical and surgical settings. Enhanced image reconstructions and displays provide a noninvasive modality for assessment of the hip in both static and dynamic automated imaging techniques. As these technologies improve and become more accessible, and as more providers become facile with ultrasound, expedient diagnosis of complex pathology without the use of CT or MRI is possible.

Improved ultrasound image quality can provide more accurate office diagnosis of multiple pathologies which are typically MRI dependent. For example, the diagnoses of acetabular labrum or synovial pathologies, proximal hamstring and gluteal tendon tears, sciatic nerve entrapments and various other periacetabular soft tissue pathologies can be readily diagnosed via ultrasound.

Contributors ER: Substantial contributions to the conception or design of the work; drafting the work and revising it critically for important intellectual content; final approval of the version to be published. ZTS: Substantial contributions to the conception or design of the work; drafting the work and revising it critically for important intellectual content; final approval of the version to be published. EA: Substantial contributions to the conception or design of the work; drafting the work and revising it critically for important intellectual content; final approval of the version to be published.

Funding The authors have not declared a specific grant for this research from any funding agency in the public, commercial or not-for-profit sectors.

Competing interests None declared.

Patient consent for publication Not required.

Provenance and peer review Commissioned; externally peer reviewed.

Data availability statement There are no data in this work.

ORCID iD

Eyal Amar http://orcid.org/0000-0001-6358-5374 


\section{REFERENCES}

1 Sharpe RE, Nazarian LN, Levin DC, et al. The increasing role of nonradiologists in performing ultrasound-guided invasive procedures. J Am Coll Radiol 2013;10:859-63.

2 Dussik KT, Möglichkeit Über die. Über die Möglichkeit, hochfrequente mechanische Schwingungen ALS diagnostisches Hilfsmittel zu verwerten. Zeitschrift für die gesamte Neurologie und Psychiatrie 1942;174:153-68.

3 Lawrence JP. Physics and instrumentation of ultrasound. Crit Care Med 2007:35:S314-22.

4 Czyrny Z. Standards for musculoskeletal ultrasound. J Ultrason 2017;17:182-7.

5 Castriota-Scanderbeg A, Micheli VD, Scarale MG, et al. Precision of sonographic measurement of articular cartilage: inter- and intraobserver analysis. Skeletal Radiol 1996;25:545-9.

6 Philippon MJ, Michalski MP, Campbell KJ, et al. An anatomical study of the acetabulum with clinical applications to hip arthroscopy. J Bone Joint Surg Am 2014;96:1673-82

7 Koski JM, Anttila PJ, Isomäki HA. Ultrasonography of the adult hip joint. Scand J Rheumatol 1989;18:113-7.

8 Sada PN, Rajan P, Jeyaseelan L, et al. Standards for ultrasonographic measurements of the hip joint in Indian adults. Skeletal Radiol 1994;23:111-2.

9 Spear IM, Lipscomb PR. Noninfectious trochanteric bursitis and Peritendinitis. Surgical Clinics of North America 1952:32:1217-24.

10 Shbeeb MI, Matteson EL. Trochanteric bursitis (greater trochanter pain syndrome). Mayo Clinic Proceedings 1996;71:565-9.

11 Connell DA, Bass C, Sykes CJ, et al. Sonographic evaluation of gluteus medius and minimus tendinopathy. Eur Radiol 2003;13:1339-47.

12 Kong A, Van der Vliet A, Zadow S. Mri and US of gluteal tendinopathy in greater trochanteric pain syndrome. Eur Radiol 2007;17:1772-83.

13 Garcia FL, Picado CHF, Nogueira-Barbosa MH. Sonographic evaluation of the abductor mechanism after total hip arthroplasty. J Ultrasound Med 2010;29:465-71.

14 Graif M, Seton A, Nerubai J, et al. Sciatic nerve: sonographic evaluation and anatomicpathologic considerations. Radiology 1991;181:405-8.

15 Ganz R, Parvizi J, Beck M, et al. Femoroacetabular impingement: a cause for osteoarthritis of the hip. Clin Orthop Relat Res 2003;417:112-20.

16 Buck FM, Hodler J, Zanetti M, et al. Ultrasound for the evaluation of femoroacetabular impingement of the CAM type. diagnostic performance of qualitative criteria and alpha angle measurements. Eur Radiol 2011;21:167-75.

17 Lerch S, Kasperczyk A, Berndt T, et al. Ultrasound is as reliable as plain radiographs in the diagnosis of cam-type femoroacetabular impingement. Arch Orthop Trauma Surg 2016:136:1437-43.

18 Robinson DJ, Lee S, Marks P, et al. Ultrasound determination of the femoral head-neck alpha angle. Ultrasound Med Biol 2018:44:495-501.

19 de Sa D, Alradwan H, Cargnelli S, et al. Extra-Articular hip impingement: a systematic review examining operative treatment of psoas, subspine, ischiofemoral, and greater trochanteric/pelvic impingement. Arthroscopy 2014;30:1026-41.

20 Larson CM, Kelly BT, Stone RM. Making a case for anterior inferior iliac spine/ subspine hip impingement: three representative case reports and proposed concept. Arthroscopy 2011;27:1732-7.

21 Amar E, Warschawski Y, Sharfman ZT, et al. Pathological findings in patients with low anterior inferior iliac spine impingement. Surg Radiol Anat 2016;38:569-75.

22 Hetsroni I, Poultsides L, Bedi A, et al. Anterior inferior iliac spine morphology correlates with hip range of motion: a classification system and dynamic model. Clin Orthop Relat Res 2013;471:2497-503.

23 Qvistgaard E, Torp-Pedersen S, Christensen R, et al. Reproducibility and inter-reader agreement of a scoring system for ultrasound evaluation of hip osteoarthritis. Ann Rheum Dis 2006;65:1613-9.

24 Moss SG, Schweitzer ME, Jacobson JA, et al. Hip joint fluid: detection and distribution at MR imaging and US with cadaveric correlation. Radiology 1998;208:43-8.
25 Bierma-Zeinstra SMA, Bohnen AM, Verhaar JA. Sonography for hip joint effusion in adults with hip pain. Ann Rheum Dis 2000;59:178-82.

26 Friedman T, Miller TT. Mr imaging and ultrasound correlation of hip pathologic conditions. Magn Reson Imaging Clin N Am 2013:21:183-94.

27 Winston P, Awan R, Cassidy JD, et al. Clinical examination and ultrasound of self-reported snapping hip syndrome in elite ballet dancers. Am J Sports Med 2007;35:118-26

28 Wahl CJ, Warren RF, Adler RS, et al. Internal coxa saltans (snapping hip) as a result of overtraining: a report of 3 cases in professional athletes with a review of causes and the role of ultrasound in early diagnosis and management. Am J Sports Med 2004;32:1302-9.

29 Long SS, Surrey DE, Nazarian LN. Sonography of greater trochanteric pain syndrome and the rarity of primary bursitis. AJR Am J Roentgenol 2013;201:1083-6.

30 Finnoff JT, Bond JR, Collins MS, et al. Variability of the Ischiofemoral space relative to femur position: an ultrasound study. Pm R 2015;7:930-7.

31 Singer $A D$, Subhawong $T K$, Jose J, et al. Ischiofemoral impingement syndrome: a metaanalysis. Skeletal Radiol 2015;44:831-7.

32 Leopold SS, Battista V, Oliverio JA. Safety and efficacy of intraarticular hip injection using anatomic landmarks. Clin Orthop Relat Res 2001;391:192-7.

33 Hoeber S, Aly A-R, Ashworth N, et al. Ultrasound-Guided hip joint injections are more accurate than landmark-guided injections: a systematic review and meta-analysis. $\mathrm{Br} J$ Sports Med 2016;50:392-6.

34 Sviggum HP, Ahn K, Dilger JA, et al. Needle echogenicity in Sonographically guided regional anesthesia. J Ultrasound Med 2013;32:143-8.

35 Jacobson J. A. Interventional techniques. In: Jacobson J, ed. Fundamentals of musculoskeletal ultrasound. 2nd edn. Philadelphia: Elsevier, Saunders, 2013: 338-69.

36 Charboneau JW, Reading CC, Welch TJ. Ct and sonographically guided needle biopsy: current techniques and new innovations. AJR Am J Roentgenol 1990;154:1-10.

37 Yoong P, Guirguis R, Darrah R, et al. Evaluation of ultrasound-guided diagnostic local anaesthetic hip joint injection for osteoarthritis. Skeletal Radiol 2012;41:981-5.

38 Payne JM, Procedures U-GH. Ultrasound-Guided hip procedures. Phys Med Rehabil Clin N Am 2016:27:607-29.

39 Blankenbaker DG, De Smet AA, Keene JS. Sonography of the iliopsoas tendon and injection of the iliopsoas bursa for diagnosis and management of the painful snapping hip. Skeletal Radiol 2006;35:565-71.

40 Williams BS, Cohen SP. Greater trochanteric pain syndrome: a review of anatomy, diagnosis and treatment. Anesth Analg 2009;108:1662-70.

41 Cohen SP, Narvaez JC, Lebovits AH, et al. Corticosteroid injections for trochanteric bursitis: is fluoroscopy necessary? A pilot study. Br J Anaesth 2005;94:100-6.

42 McEvoy JR, Lee KS, Blankenbaker DG, et al. Ultrasound-Guided corticosteroid injections for treatment of greater trochanteric pain syndrome: greater trochanter bursa versus subgluteus medius bursa. AJR Am J Roentgenol 2013;201:W313-7.

43 Finnoff JT, Hurdle MFB, Smith J. Accuracy of ultrasound-guided versus Fluoroscopically guided Contrast-Controlled piriformis injections. J Ultrasound Med 2008;27:1157-63.

44 Fowler IM, Tucker AA, Weimerskirch BP, et al. A randomized comparison of the efficacy of 2 techniques for piriformis muscle injection: ultrasound-guided versus nerve stimulator with fluoroscopic guidance. Reg Anesth Pain Med 2014;39:126-32.

45 Wisniewski SJ, Hurdle M, Erickson JM, et al. Ultrasound-Guided ischial bursa injection: technique and positioning considerations. Pm R 2014;6:56-60.

46 Van Mieghem IM, Boets A, Sciot R, et al. Ischiogluteal bursitis: an uncommon type of bursitis. Skeletal Radiol 2004;33:413-6.

47 Nwawka OK, Meyer R, Miller TT. Ultrasound-Guided subgluteal sciatic nerve perineural injection: report on safety and efficacy at a single institution. J Ultrasound Med 2017;36:2319-24.

48 Rosales J, García N, Rafols C, et al. Perisciatic ultrasound-guided infiltration for treatment of deep gluteal syndrome: description of technique and preliminary results. J Ultrasound Med 2015;34:2093-7. 